\title{
Three-dimensional Phase-Field Modeling of Spinodal Decomposition in Constrained Films
}

\author{
D. J. Seol ${ }^{* * * *}$, S. Y. Hu**, Y. L. Li ${ }^{* *}$, J. Shen ${ }^{\dagger}$, K. H. Oh*, and L. Q. Chen** \\ * School of Materials Science and Engineering \\ Research Institute of Advanced Materials, Seoul National University \\ San 56-1 Shillim-dong, Gwanak-gu, Seoul 151-742, Korea \\ **Department of Materials Science and Engineering, The Pennsylvania State University \\ University Park, PA 16802, USA \\ 'Department of Mathematics, Purdue University, West Lafayette, IN 47907, USA
}

\begin{abstract}
The morphological evolution during spinodal decomposition in binary alloy thin films elastically constrained by substrates is studied. Elastic solutions, derived for both elastically isotropic and anisotropic thin films subject to mixed stress-free and constraint boundary conditions, are employed in a three-dimensional phasefield model to investigate the effect of coherency strain and substrate constraint on microstructural evolution. The temporal evolution of the Cahn-Hilliard equation under thin film boundary conditions is solved with a semi-implicit Fourier-spectral method. The phase separation with coherency strain in an elastically anisotropic film shows the behavior of surface-directed spinodal decomposition driven by the elastic energy effect. Negative elastic anisotropy in the cubic alloy causes the alignment of the phases along $\langle 100\rangle$ elastically soft directions.
\end{abstract}

Keywords : spinodal decomposition, thin film, strain energy, anisotropy, phase-field model

\section{INTRODUCTION}

In spinodal decomposition, phase separation occurs by the spontaneous amplification of compositional fluctuations when a homogeneous phase is quenched from the one-phase region into the unstable part of a miscibility gap. Because new two phases form by a continuous process in the spinodal decomposition, the two phases start out to be coherent with one another and the lattice initially remains continuous [1]. The formation of coherent microstructures generates elastic strain energy whose magnitude depends on the degree of lattice mismatch, the elastic properties of each phase, and the spatial distributions of coherent domains [2]. Since Cahn first introduced the elastic energy term in the expression of the total free energy of the system [3], there have been a number of studies on the thermodynamics of coherent systems including the interaction of composition and stress in crystalline solids [2,4-8].

Formulations for coherent decomposition have recently been developed especially for confined geometry such as thin films or plates in accordance with recent interest in the thin film applications of materials [9-11]. When one considers binary mixtures $(\mathrm{AB})$ confined in thin film geometry, the growth of A-rich and B-rich domains and their morphology are distinctly modified compared to those of bulk [12]. Thin film geometry includes the substrate, the film, the free surface of the film and the film/substrate interface. The film is mechanically constrained by the substrate. The average lattice mismatch and the difference in thermal expansion coefficient between the film and substrate cause strain energy in addition to thecoherency strain energy. Leo et al. [13] developed essentially a 1-dimensional formulation for the spinodal decomposition of stressed thin films on compliant substrates. In their model the substrate and film are elastically isotropic, and the substrate affects the stress state in the film through both the average misfit and the bending stiffness of the layered system.

In this paper, we extend the phase-field approach with a Cahn-Hillard-type diffusion equation to describe a 3-dimensional morphological evolution during spinodal decomposition and coarsening in a thin film attached to a substrate at constant temperature. The film is assumed to be a binary

This article is based on a presentation made in the 2002 Korea-US symposium on the "Phase Transformations of Nano-Materials," organized as a special program of the 2002 Annual Meeting of the Korean Institute of Metals and Materials, held at Yonsei University, Seoul, K orea on October 25-26, 2002. 
(AB) alloy with two equilibrium phases. The substrate doesnt interact diffusionally with the film. We also assume the substrate to be sufficiently thick compared to the film thickness, so that deformation is homogeneous. Elastic solutions are derived for both elastically anisotropic and isotropic thin films subject to the mixed stress-free and constraint boundary conditions. These thin film boundary conditions have been successfully applied to the phase-field simulation of the formation of martensitic domains [14-16]. Elastic solutions from thin film boundary conditions are employed in a 3dimensional phase-field model.

\section{PHASE-FIELD MODEL}

\subsection{Kinetic evolution equation}

The temporal evolution of the composition field is described by the Cahn-Hilliard-type diffusion equation given by

$$
\frac{\partial X}{\partial t}=\nabla \cdot\left[M \nabla\left(\frac{\delta F}{\delta X}\right)\right]
$$

where $X$ is the composition of species 2 (mole or atom fraction) and $F$ is the total free energy of the system. If we assume that the atomic mobilities of species 1 and 2 are equal, the mobility, $M$, is given by [17].

$$
M=\frac{D X(1-X)}{k_{B} T}
$$

where $D$ is the diffusion coefficient, $k_{B}$ the Boltzmann constant and $T$ the temperature. The mobility is further simplified by assuming that the factor is a constant given by where $X_{0}$ is the overall composition.

Because the total free energy in Eq. 1 includes the bulk chemical free energy, interfacial energy and elastic energy, we have

$$
\frac{\partial X}{\partial t}=\nabla \cdot\left[\frac{D X_{0}\left(1-X_{0}\right)}{k_{B} T} \nabla\left(\frac{d f(X)}{d x}-\alpha \nabla^{2} X+\mu_{e l}\right)\right]
$$

where $f$ is the bulk chemical free energy density, $\alpha$ the gradient energy coefficient which is related to the interfacial energy, and $\mu_{e l}$ the elastic potential which is the derivative of an elastic energy with respect to the composition.

Using reduced variables we have the dimensionless form of the kinetic equation:

$$
\frac{\partial X\left(\bar{r}^{*}, t^{*}\right)}{\partial t^{*}}=\left(\nabla^{*}\right)^{2}\left(\frac{d f^{*}(X)}{d X}-\alpha^{*}\left(\nabla^{*}\right)^{2} X+\mu_{e l}^{*}\right)
$$

where $r^{*}=r / l_{0}, t^{*}=D t X_{0}\left(1-X_{0}\right) / l_{0}^{2}, \nabla^{*}=l_{0}^{2} \nabla, f^{*}=f /\left(k_{B} T\right)$, $\alpha^{*}=\alpha /\left(k_{B} T l_{0}^{2}\right)$ and $\mu_{e l}^{*}=\mu_{e l} /\left(k_{B} T\right)$, where $l_{0}$ is the grid spacing.

The chemical free energy density, $f$, is described by a fourth order polynomial reduced to have minima at equilibrium compositions of \pm 1.0 as follows:

$$
f^{*}(X)=-\frac{1}{2} X^{2}+\frac{1}{4} X^{4}
$$

\subsection{Elastic energy in constrained films}

If we assume variation in the stress-free lattice parameter, $a$, the composition is linear, i.e. (it obeys the Vegard's law) the local stress-free strain caused by compositional inhomogeneity is given by [17].

$$
\varepsilon_{i j}^{0}(\bar{r})=\varepsilon_{0} \delta X(\bar{r}) \delta_{i j}
$$

where is the compositional expansion coefficient of the lattice parameter and $\delta_{i j}$ the Kronecker-Delta function.

In the linear elasticity, the stress $\sigma_{i j}$ is related to the elastic strain by Hookes law:

$$
\sigma_{i j}=c_{i j k l}\left(\varepsilon_{k l}(\bar{r})-\varepsilon_{k l}^{0}(\bar{r})\right)
$$

where $c_{i j k l}$ is the elastic stiffness which gives the crystal elastic constants and $\varepsilon_{k l}(\bar{r})$ is the total strain measured with respect to a reference lattice. The effect of composition on the elastic constants is neglected. The total strain can be separated into homogeneous and heterogeneous strains [2]:

$$
\varepsilon_{i j}(\bar{r})=\bar{\varepsilon}_{i j}+\delta \varepsilon_{i j}(\bar{r})
$$

where the heterogeneous strain, $\delta \varepsilon_{i j}(\bar{r})$, is defined so that

$$
\int_{v} \delta \varepsilon_{i j}(\bar{r}) d^{3} r=0
$$

The homogeneous strain is the uniform macroscopic strain characterizing the macroscopic shape and volume change associated with the total strain. The relationship between the strain and displacement gives the following equation:

where $u_{i}(\bar{r})$ denotes the $i$ th component of displacement.

The mechanical equilibrium equations with respect to elastic displacements are expressed as

$$
\frac{\partial \sigma_{i j}}{\partial r_{j}}=0
$$

where $r_{j}$ is the jth component of $\bar{r}$. The stress-free boundary condition at the top surface of a film is given by

$$
\left.\sigma_{i 3}\right|_{x_{3}=h_{f}}=0
$$

where $h_{f}$ is the film thickness along $z$ direction as shown in Fig. 1. Since the elastic perturbation resulting from the heterogeneous strain disappears in the substrate far from the filmsubstrate interface, the following condition is used to describe the constraint of the substrate. 


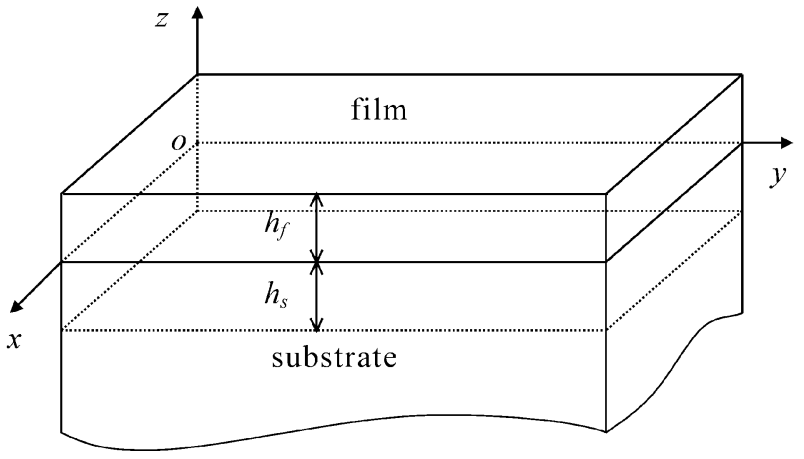

Fig. 1. Schematic illustration of a thin film coherently constrained by a substrate.

$$
\left.u_{i}\right|_{x_{3}=-h_{s}}=0
$$

where $h_{s}$ is the distance from the film-substrate interface into the sufficiently thick substrate, beyond which the elastic deformation is ignored (see Fig. 1).

To solve Eqs. 11-13, two elastic solutions are superposed [15]. The first solution is from Khachaturyans microelasticity theory [2] within $0<x_{3}<h_{f}$ and the second is from the elastic solution in an infinite plate of thickness $h_{f}+h_{s}$, satisfying the two boundary conditions. Consequently, the elastic energy in the constrained thin film can be calculated from Eqs. 7, 8 and 10 .

\section{3-D SIMULATION OF THE SPINODAL DECOMPOSITION}

Taking the Fourier-transform of Eq. 4, we have the temporal evolution of the composition wave amplitude, $X\left(\bar{g}^{*}, t^{*}\right)$,

$$
\frac{\partial X\left(\bar{g}^{*}, t^{*}\right)}{\partial t^{*}}=-\left(g^{*}\right)^{2}\left[\left(\frac{d f^{*}(X)}{d X}\right)_{\bar{g}^{*}}+\alpha^{*}\left(g^{*}\right)^{2} X\left(\bar{g}^{*}, t^{*}\right)+\mu_{e l}^{*}\left(\bar{g}^{*}\right)\right]
$$

where $g^{*}$ is the magnitude of $\bar{g}^{*}$, a vector in the Fourier space, $X\left(\bar{g}^{*}, t^{*}\right),\left(d f^{*}(X) /(d X)\right)_{\bar{g}^{*}}$ and $\mu_{e l}^{*}\left(\bar{g}^{*}\right)$ are the Fourier transforms of $X\left(\bar{r}^{*}, t^{*}\right),\left(d f^{*}(X) /(d X)\right)_{\bar{r}^{*}}$, and $\mu_{e l}^{*}\left(\bar{r}^{*}\right)$, respectively. The above equation can be efficiently solved using a semiimplicit method [17,18]. More precisely,

$$
\begin{aligned}
& X^{n+1}\left(\bar{g}^{*}\right)= \\
& \frac{\frac{4 X^{n}\left(\bar{g}^{*}\right)-X^{n-1}\left(\bar{g}^{*}\right)}{3}-\frac{2}{3} \Delta t^{*}\left(g^{*}\right)^{2}\left[2\left(\mu^{*}\left(\bar{g}^{*}\right)\right)^{n}-\left(\mu^{*}\left(\bar{g}^{*}\right)\right)^{n-1}\right]}{1+\frac{2}{3} \Delta t^{*} \alpha^{*}\left(g^{*}\right)^{4}}
\end{aligned}
$$

where $\mu^{*}=d f^{*}(X) / d X+\mu_{e l}^{*}$ and $\Delta t^{*}$ is the time step for integration.

For time discretization, a second order backward differ- ence scheme is applied. For spatial discretization, a Fourierspectral method is used in the $x$ and $y$ direction, and since the zero-flux boundary conditions at the film surface and film/ substrate interface cannot be satisfied by a Fourier expansion, a second-order finite difference method with FFT (Fast

Fourier Transform) is used in the $z$-direction.

In the computer simulation, discrete grid points are used where $h_{f}=32 l_{0}$, and periodic boundary conditions are applied along the $x_{1}$ and $x_{2}$ axes. The dimensionless grid spacing in real space is chosen to be $\Delta x_{1} / l_{0}=\Delta x_{2} / l_{0}=\Delta x_{3} / l_{0}=1.0$ and $\Delta t^{*}$ is chosen to be 0.05 . The dimensionless gradient energy coefficient, $\alpha^{*}$, is chosen to be 0.6 with which the diffuse interface includes about 2 or 3 grid points. The interface energy between the decomposed phases is assumed to be isotropic. The overall reduced composition used in this study is an off-critical composition of -0.3. A small compositional fluctuation is applied to initiate the decomposition process by random number generation at the initial stage. The elastic constants used for the film are $C_{11}=300, C_{12}=100, C_{44}=100$ for isotropic cubic material, and $C_{11}=300, C_{12}=200, C_{44}=100$ for anisotropic cubic material, all in units of $N_{V} k_{B} T$ where $N_{V}$ is the number of atoms per unit volume. Same elastic constants are used for the substrate. Note that the anisotropy given by $\left(C_{11}-C_{12}-2 C_{44}\right) C_{44}$ is negative. The film is assumed to be coherent with the substrate and the degree of substrate constraint is measured by the mismatch strain between the film and the substrate, $\bar{\varepsilon}_{i j}(i, j=1,2)$.

\section{RESULTS AND DISCUSSION}

The local stress field in the thin film is caused by local compositional inhomogeneity with coherent interfaces between decomposed phases. If we assume the atomic sizes of $\mathrm{A}$ and $\mathrm{B}$ atoms in the film are the same and that there is no elastic constraint on the film by the substrate, the spinodal decomposition is driven by bulk chemical free energy and interface energy without elastic energy. Fig. 2 shows the temporal evolution of the phases during the spinodal decomposition in thin film without elastic energy. The solid region corresponds to the higher concentration (B-rich) and the bottom plate is the substrate. Decomposition occurs homogeneously throughout the film from the initial stage. From the 3-dimensional observation, the B-rich phases are interconnected randomly, i.e., without any preferred direction of interconnection or layer formation. If the film thickness becomes very thin, the microstructure is expected to show little change along the $z$-direction. The limiting case is a 2-dimensional microstructure on the $x y$ plane.

Fig. 3 shows the temporal evolution of the phases during the spinodal decomposition of an elastically isotropic material whose compositional expansion coefficient of the lattice parameter is 0.1 . The initial decomposition starts at the film surface where it is easier to relieve the local stress field 


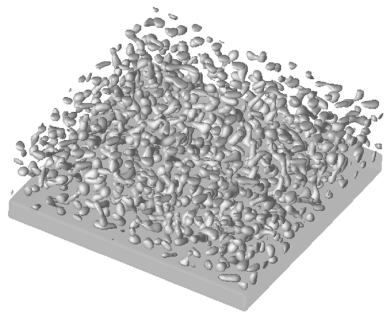

(a) $t^{*}=5$

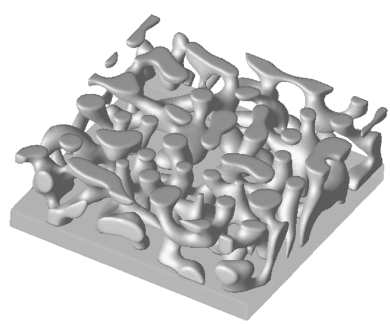

(c) $t^{*}=60$

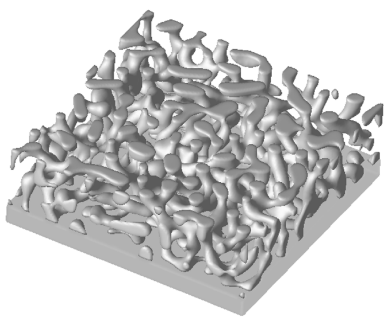

(b) $t^{*}=20$

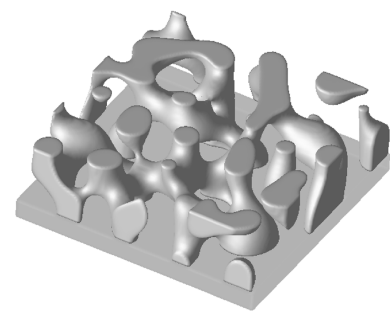

(d) $t^{*}=200$
Fig. 2. Temporal evolution of the phases during the spinodal decomposition in thin film without the elastic energy.

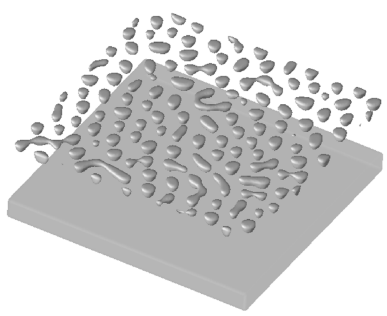

(a) $t^{*}=10$

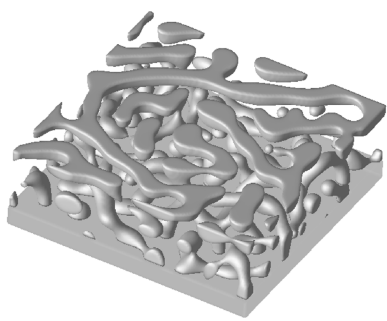

(c) $t^{*}=60$

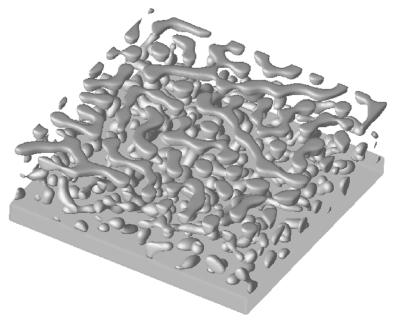

(b) $t^{*}=20$

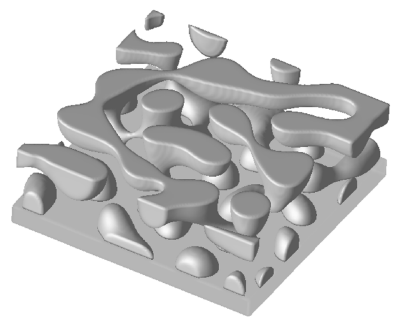

(d) $t^{*}=200$
Fig. 3. Temporal evolution of the phases during the spinodal decomposition in an elastically isotropic film.

accompanied by the decomposition. Compared to Fig. 2, the decomposed phases have a slight tendency to align parallel to the $x y$-plane due to the initial layer formation at the film surface.

Fig. 4 shows the temporal evolution of the phases during the spinodal decomposition of an elastically anisotropic material whose compositional expansion coefficient of the lattice parameter is 0.1 . From the early stage of decomposition at the film surface, the decomposed phases have a tendency to align along $\langle 100\rangle$ directions. For a cubic alloy with

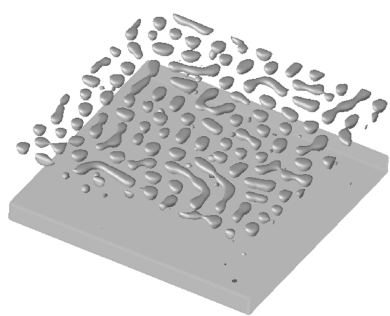

(a) $t^{*}=10$

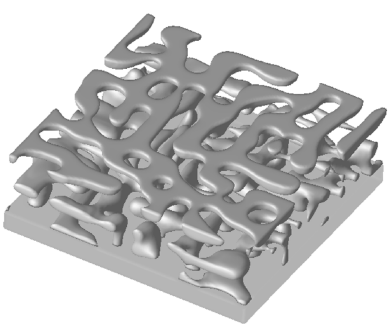

(c) $t^{*}=60$

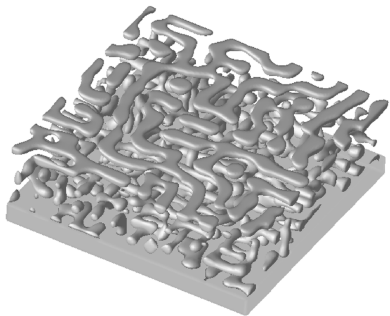

(b) $t^{*}=20$

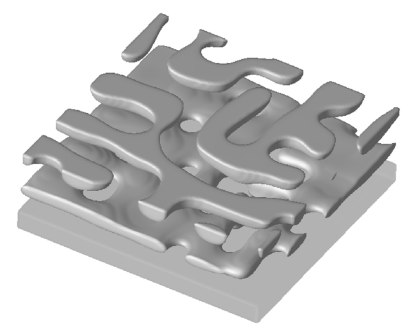

(d) $t^{*}=200$
Fig. 4. Temporal evolution of the phases during the spinodal decomposition in an elastically anisotropic film.

negative elastic anisotropy, $<100>$ directions are elastically soft directions and morphological alignment along those directions reduces the elastic energy [18]. As the B-rich domain coarsens, the morphology not only shows a strong <100> alignment in the $x y$-plane, but also shows a layered structure along the $z$-direction. The B-rich phase seldom interconnects along the $z$-direction at the composition of -0.3 because of the gradual decomposition process that will be explained in the next figure.

The formation of the layered structure can be effectively observed in a 2-dimensional $x z$-section by increasing the

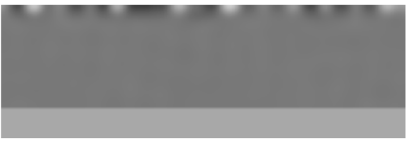

(a) $t^{*}=4.0$

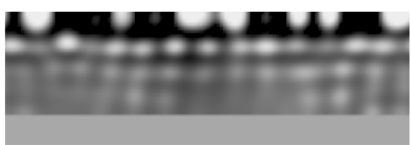

(c) $t^{*}=8.5$

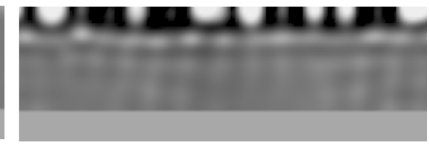

(b) $t^{*}=6.5$

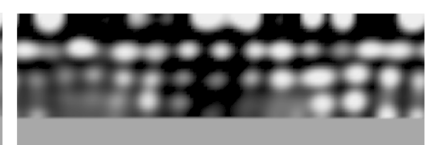

(d) $t^{*}=11.5$

Fig. 5. Sequential formation of the layered structure observed in the $x z$-section at (a) $t^{*}=4.0$, (b) $t^{*}=6.5$, (c) $t^{*}=8.5$, and (d) $t^{*}=11.5$.

time as shown in Fig. 5. The bright and dark regions correspond to the B-rich and A-rich regions, respectively. The phase separation starts at the film surface first and the volume fraction of the B-rich phase at the surface is much higher than the equilibrium value. It develops a compressive stress field. So the next layer is almost A-rich where the B 

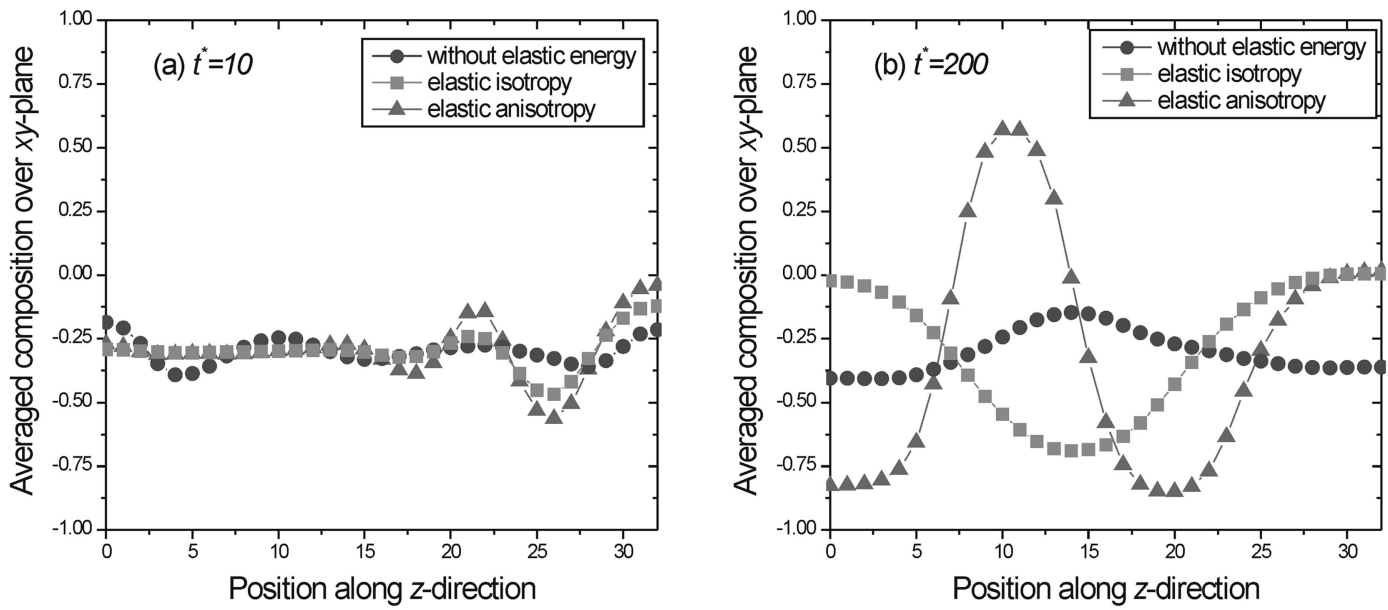

Fig. 6. Profiles of averaged compositions over the $x y$-plane with respect to the position along the $z$-direction from the film/substrate interface to the film surface at (a) $t^{*}=10$ and (b) $t^{*}=200$.

component is depleted. The B-rich region is hard to form right beneath the surface layer because the compressive stress field overlaps. The layer forming process propagates gradually with time from the film surface to the film/substrate interface. As the layered structure forms, the alternating compressive and tensile stress fields develop without causing a macroscopic displacement in the $x$ or $y$-direction. The phase separation procedure observed in Fig. 5 can be thought of as a kind of surface-directed spinodal decomposition [19], driven by the elastic effect.

Figs. 6(a) and (b) show the profiles of averaged compositions over the $x y$-plane with respect to the position along the $z$-direction from the film/substrate interface to the film surface. When $t^{*}=10$ in Fig. 6(a), the coherency strain energy causes compositional fluctuations at the film surface first and the compositions near the film/substrate interface are still homogeneous in both the elastically isotropic and anisotropic materials. As to the film without elastic energy, the compositional fluctuation exists throughout the film thickness. When $t^{*}=200$ in Fig. 6(b), all composition profiles fluctuate. The elastically anisotropic material, especially, shows the largest fluctuation along the z-direction forming the layered structure. The coherency strain energy and elastic anisotropy increase the tendency of the layer formation for the given condition studied here.

\section{CONCLUSIONS}

A three-dimensional phase-field model is employed to study the morphological evolution during the spinodal decomposition in binary alloy thin films elastically constrained by the substrates. Without coherency strain, the compositional fluctuation is homogeneous in the whole film from the initial stage of the spinodal decomposition. When the coherency strain is introduced, the phase separation fol- lows the mechanism of surface-directed spinodal decomposition, driven by the elastic energy effect. The resultant microstructure in an elastically anisotropic film is an alternately aligned layer structure along the $z$-direction parallel to the film surface. Due to the negative elastic anisotropy in the cubic alloy, decomposed phases align along elastically soft $<100\rangle$ directions to reduce the elastic energy.

\section{ACKNOWLEDGMENT}

The authors are grateful for the financial support for this research of the National Research Laboratory Project through the Ministry of Science and Technology, and of the US National Science Foundation under grant numbers DMR01-22638 and DMS-0074283.

\section{REFERENCES}

1. J. W. Cahn, Trans. AIME 242, 166 (1968).

2. A. G. Khachaturyan, Theory of Structural Transformations in Solids, Wiley, New York (1983).

3. J. W. Cahn, Acta metall. 10, 907 (1962).

4. J. W. Cahn and F. Larché, Acta metall. 32, 1915 (1984).

5. F. C. Larché and J. W. Cahn, Acta metall. 33, 331 (1985).

6. W. C. Johnson and P. W. Voorhees, Metall. Trans. A 18, 1213 (1987).

7. W. C. Johnson, Metall. Trans. A 18, 1093 (1987).

8. D. Orlikowski, C. Sagui, A. Somoza, and C. Roland, Phys. Rev. B 59, 8646 (1999).

9. F. C. Larché and J. W. Cahn, Acta metall. mater. 40, 947 (1992).

10. J. W. Cahn and R. Kobayashi, Acta metall. mater. 43, 931 (1995).

11. G. Abadias, A. Marty, and B. Gilles, Acta mater. 46, 6403 (1998). 
12. K. Binder, J. Non-Equilib. Thermodyn. 23, 1 (1998).

13. P. H. Leo and W. C. Johnson, Acta mater. 49, 1771 (2001).

14. Y. L. Li, S. Y. Hu, Z. K. Liu, and L. Q. Chen, Appl. Phys. Lett. 78, 3878 (2001).

15. Y. L. Li, S. Y. Hu, Z. K. Liu, and L. Q. Chen, Acta mater. 50, 395 (2002).

16. D. J. Seol, S. Y. Hu, Y. L. Li, L. Q. Chen, and K. H. Oh,
Mater. Sci. Forum 408-412, 1645 (2002).

17. S. Y. Hu and L. Q. Chen, Acta mater. 49, 1879 (2001).

18. L. Q. Chen and J. Shen, Comput. Phys. Commun. 108, 147 (1998).

19. S. Puri and H. L. Frisch, J. Phys.: Condens. Matter 9, 2109 (1997). 